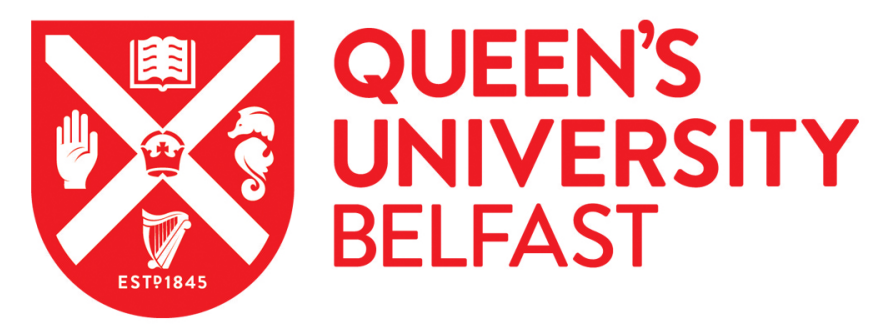

\title{
Weight-lowering effects of glucagon-like peptide-1 receptor agonists and detection of breast cancer among obese women with diabetes
}

\author{
Santella , C., Yin , H., Hicks, B., Yu, O., Bouganim , N., \& Azoulay , L. (2020). Weight-lowering effects of \\ glucagon-like peptide-1 receptor agonists and detection of breast cancer among obese women with diabetes. \\ Epidemiology. https://doi.org/10.1097/EDE.0000000000001196
}

\section{Published in:}

Epidemiology

\section{Document Version:}

Peer reviewed version

Queen's University Belfast - Research Portal:

Link to publication record in Queen's University Belfast Research Portal

Publisher rights

Copyright 2019 Lippincott, Williams \& Wilkins. This work is made available online in accordance with the publisher's policies. Please refer to any applicable terms of use of the publisher.

\section{General rights}

Copyright for the publications made accessible via the Queen's University Belfast Research Portal is retained by the author(s) and / or other copyright owners and it is a condition of accessing these publications that users recognise and abide by the legal requirements associated with these rights.

Take down policy

The Research Portal is Queen's institutional repository that provides access to Queen's research output. Every effort has been made to ensure that content in the Research Portal does not infringe any person's rights, or applicable UK laws. If you discover content in the Research Portal that you believe breaches copyright or violates any law, please contact openaccess@qub.ac.uk. 


\section{ABSTRACT}

Background: It has been proposed that the weight loss associated with glucagon-like peptide-1 receptor agonists (GLP-1 RAs) may improve the detection of breast cancer in patients undergoing this treatment. Thus, this study aimed to determine whether the weightlowering effects of GLP-1 RAs is associated with an increased detection of breast cancer among obese women with type 2 diabetes.

Methods: Using the United Kingdom Clinical Practice Research Datalink, we conducted a propensity score-matched cohort study among female obese patients with type 2 diabetes newly-treated with antidiabetic drugs between 1 January 2007 and 31 January 2018. New users of GLP-1 RAs $(n=5,510)$ were matched to new users of second- to third-line noninsulin antidiabetic drugs $(\mathrm{n}=5,510)$. Time-dependent Cox proportional hazards models were used to estimate hazard ratios (HRs) and 95\% confidence intervals (CIs) of breast cancer associated with different GLP-1 RA maximal weight loss categories (<5\%, 5\%-10\%, >10\%). Results: Breast cancer incidence gradually increased with GLP-1 RA maximal weight loss categories, with the highest HR observed for patients achieving at least $10 \%$ weight loss (HR: 1.77, 95\% CI: 1.12, 2.81). In secondary analyses, the HR for $>10 \%$ weight loss was highest in the 2-3 years since treatment initiation (HR: 2.90, 95\% CI: 1.21, 6.92).

Conclusions: In this population-based study, the detection of breast cancer gradually increased with GLP-1 RA weight loss categories, particularly among those achieving $>10 \%$ weight loss. These results suggest that significant weight loss with GLP-1 RAs may improve the detection of breast cancer among obese patients with type 2 diabetes. 


\section{INTRODUCTION}

Glucagon-like peptide-1 receptor agonists (GLP-1 RAs) are second- to third-line incretin-based drugs used in the treatment of type 2 diabetes. ${ }^{1}$ These drugs have been shown to have cardiovascular benefits, while also inducing significant weight loss through appetite suppression. ${ }^{2-4}$ Despite these favorable effects, there are concerns that GLP-1 RAs may increase the risk of certain malignancies, including breast cancer. ${ }^{5}$

The association between GLP-1 RAs and breast cancer is controversial. While certain GLP-1 RAs have been associated with an excess number of breast cancer events in premarketing trials, ${ }^{5}$ subsequent cardiovascular outcome trials among patients with type 2 diabetes $^{6-8}$ and weight management trials among obese patients ${ }^{9}$ have generated conflicting findings. However, these trials were of short durations (less than 4 years) and were not designed to assess breast cancer as a safety endpoint. ${ }^{6,7,9}$ Moreover, while no overall increased risk of breast cancer was reported in the LEADER trial, ${ }^{6}$ a post-hoc analysis revealed a transient imbalance of breast cancer events with liraglutide between 1.5 and 2.5 years after randomization. ${ }^{10}$ This pattern was mirrored in the two observational studies investigating this possible association, ${ }^{11,12}$ where GLP-1 RAs appeared to be associated with a transient increased risk of breast cancer between two and three years of use.

While a tumor promoter effect may explain these patterns, ${ }^{13}$ an alternate hypothesis involves the rapid weight loss induced by GLP-1 RAs. ${ }^{14}$ Particularly, GLP-1 RAs are preferentially prescribed to obese women, ${ }^{15,16}$ a population less likely to participate in breast cancer screening programs. ${ }^{17-20}$ Thus, the weight loss induced by GLP-1 RAs may be facilitating the detection of breast masses and leading to earlier diagnosis of prevalent disease in this under-screened population. 9,10,14,21 To our knowledge, no study has been conducted to investigate this hypothesis. Therefore, the objective of this population-based study was to determine whether the rapid weight loss induced by GLP-1 RAs is associated with an increased detection of breast cancer among obese women with type 2 diabetes. 


\section{METHODS}

\section{Data Source}

This study was conducted using the United Kingdom (UK) Clinical Practice Research Datalink (CPRD), using the GOLD and Aurum datasets. ${ }^{22}$ The CPRD is a large primary care database that is broadly representative of the general UK population in terms of age, sex, and ethnicity ${ }^{23}$ The CPRD uses the Read code and SNOMED classifications for medical diagnoses and procedures, and a coded dictionary based on the UK Prescription Pricing Authority Dictionary for drug prescriptions. ${ }^{23}$ Recording of medical diagnoses in the CPRD have been shown to be valid and of high quality. ${ }^{24}$ Furthermore, breast cancer diagnoses have been shown to be well recorded and highly concordant (>90\%) with those recorded in the UK National Cancer Data Repository. ${ }^{25-27}$

The study protocol (\#19_014A2) was approved by the Independent Scientific Advisory Committee of the CPRD and the Research Ethics Board of the Jewish General Hospital, Montreal, Canada.

\section{Study Population}

We identified a base cohort of all female patients, at least 40 years of age, newlytreated with a non-insulin antidiabetic drug (including metformin, sulfonylureas, prandial glucose regulators, thiazolidinediones, acarbose, dipeptidylpeptidase-4 (DPP-4) inhibitors, GLP-1 RAs, and sodium-glucose co-transporter-2 inhibitors) between 1 January 1988 and 31 January 2018. All patients were required to have at least one year of registration in the CPRD before their first prescription. Patients prescribed insulin at any time prior to their first noninsulin antidiabetic prescription were excluded, as these likely represented those with advanced type 2 diabetes. Finally, patients previously diagnosed with polycystic ovary syndrome were excluded, as this is another indication for metformin. The construction of a 
base cohort allowed us to obtain a complete treatment history of patients included in this study, and thus limiting the possibility of left truncation.

\section{Propensity Score-Matched Approach}

Using the base cohort defined above, we conducted a sequential, propensity scorematched cohort analysis. ${ }^{28-30}$ For this approach, we identified new users of GLP-1 RAs and new users of a comparator drug at each calendar month of the study period, starting 1 January 2007 (the year the first GLP-1 RA was introduced in the UK market). This generated a total of 126 sequential GLP-1 RA-comparator cohorts over the study period. Patients in the comparator drug group consisted of new users of a second- to third-line non-insulin antidiabetic drug. This group was composed of patients initiating, for the first time in their treatment history, DPP-4 inhibitors, thiazolidinediones, prandial glucose regulators, acarbose, sodium-glucose cotransporter-2 inhibitors, combination of oral antidiabetic drugs, or switching to or adding-on an antidiabetic drug after failure with metformin or sulfonylureas in monotherapy.

We then applied exclusion criteria at the time of entry into each of the sequential cohorts. We excluded patients with a body mass index $(\mathrm{BMI})<30 \mathrm{~kg} / \mathrm{m}^{2}$ (last measure before cohort entry), those with a previous diagnosis of breast cancer (including malignant and in situ carcinoma at any time before cohort entry), and those with insulin prescriptions before or at cohort entry (as this represents a last-line therapy that has been associated with weight gain). Finally, we excluded patients with less than one year of follow-up in order to take into account a lag period from treatment initiation to achieving maximal weight loss (which occurs around 6 months after GLP-1 RA initiation ${ }^{6}$ ) and to the eventual recording of breast cancer. 
We estimated the predicted probabilities of receiving a GLP-1 RA versus a comparator drug using conditional logistic regression, with calendar month (i.e., month of drug initiation) as a stratification variable, conditional on the potential confounders listed below. Each GLP-1 RA user was then matched, without replacement, to one patient initiating a comparator drug on the same calendar month and propensity score using a greedy matching algorithm with a maximum caliper of $0.01 .{ }^{29}$ Importantly, GLP-1 RA users were individually matched to comparator drug users, provided that they had never used the comparator drug in their entire treatment history. Thus, both exposure groups were naïve to the drugs in question, using the entire treatment history as a washout period (i.e., this washout period ranged between a minimum of one year up to 31 years). Finally, patients included in the comparator group later switching to a GLP-1 RA during follow-up were allowed to contribute to the GLP-1 RA group and be matched to a comparator drug at the time of the switch using the same procedure detailed above.

\section{Potential Confounders}

The propensity score model considered the following variables measured at cohort entry: age, alcohol-related disorders (including alcoholism, alcoholic cirrhosis of the liver, alcoholic hepatitis, and hepatic failure), BMI (30-34.9 kg/m², 35-39.9 kg/m², 40-44.9 kg/m², $\geq 45 \mathrm{~kg} / \mathrm{m}^{2}$ ), and smoking status. It also included proxies for diabetes severity, including hemoglobin A1C (last laboratory result before cohort entry), duration of treated diabetes (defined as the time between cohort entry and first non-insulin antidiabetic prescription), macrovascular (ischemic stroke, myocardial infarction, peripheral vascular disease) and microvascular (nephropathy, neuropathy, retinopathy) complications of diabetes (all measured at any time before cohort entry). We also considered previous cancer (excluding non-melanoma skin cancer), previous oophorectomy, use of statins, and hormone 
replacement therapy. Finally, the model included markers of health-seeking behaviors in the year before cohort entry, including uptake of cancer screening (colorectal cancer screening, mammography) and influenza vaccination. Age and duration of treated diabetes were modeled as continuous variables using restricted cubic spline models to account for possible non-linear relations with the outcome. ${ }^{31}$

\section{Exposure Definition}

For the primary analysis, we used a modified intention-to-treat approach. For this approach, patients were assumed exposed starting one year after their first prescription until an incident diagnosis of breast cancer, or censored upon initiation of insulin, switching from a comparator drug to a GLP-1 RA (as switchers can contribute to the GLP-RA group), death from any cause, end of registration with the general practice, or end of the study period (31 January 2019), whichever occurred first. This approach assumes an irreversible effect of the exposure on the outcome (i.e., patients remain at risk of breast cancer even after treatment discontinuation). Censoring at the time of a switch dealt with the potential impact of crossovers on the point estimates.

In a secondary analysis, we used an as-treated approach where patients were followed while continuously exposed to the study drugs. Continuous exposure was defined by consecutive prescriptions overlapping each other and using a one-year grace period in the event of non-overlap (to account for possible diagnostic delays). This approach treats breast cancer as an acute outcome, thus assuming that breast cancer detection occurs while patients are exposed and relatively soon after treatment initiation (i.e., the rapid weight loss induced by GLP-1 RAs leads to the detection of breast masses and subsequent diagnosis of breast cancer). 


\section{Statistical Analysis}

Incidence rates of breast cancer with 95\% confidence intervals (CIs), based on the Poisson distribution, were calculated for the GLP-1 RA and comparator groups. Maximal weight loss associated with the use of GLP-1 RAs was modeled as a time-varying variable, which allowed patients to move between weight change categories during the follow-up period. Maximal weight loss was defined as the highest percent weight change ([baseline measurement - post-treatment measurement]/baseline measurement x 100\%) achieved at a given risk set (i.e., date of event; see eFigure 1). Patients with a baseline weight measurement and no subsequent measurements were assumed to have achieved no weight loss. Thus, GLP-1 RA exposed person-time was classified into one of five categories: $<5 \%$, $5 \%-10 \%,>10 \%$, no weight cange, and weight gain. Cumulative incidence curves of breast cancer were constructed for each exposure group. Time-dependent Cox proportional hazard models were used to estimate hazard ratios (HRs) and 95\% CIs of breast cancer, comparing GLP-1 RA maximal weight loss categories (<5\%, 5\%-10\%, >10\%), with the comparator drugs. We also modelled maximal weight loss as a continuous variable using a restricted cubic spline model with five knots, which produced a smooth risk function. ${ }^{32}$ Finally, we calculated incidence rates for mammography and referrals for diagnostic imaging or signs and symptoms of breast cancer across the weight loss categories over the follow-up period.

\section{Secondary Analyses}

We performed three secondary analyses. First, we assessed whether the association varied by GLP-1 RA molecule (liraglutide and exenatide). Second, we investigated whether the association varied by time since initiation of GLP-1 RAs, estimating HRs for three predefined duration categories: $\leq 2$ years, $2-3$ years, and $>3$ years; these were chosen because of the patterns observed in previous studies. ${ }^{10-12}$ Finally, we assessed effect measure 
modification by BMI by including an interaction term between the GLP-1 RA weight loss categories and baseline BMI ( $<40 \mathrm{~kg} / \mathrm{m}^{2}$ versus $\left.\geq 40 \mathrm{~kg} / \mathrm{m}^{2}\right)$.

\section{Sensitivity Analyses}

We conducted five sensitivity analyses to assess the robustness of our findings. First, to account for potential exposure misclassification, exposure was redefined by requiring at least four GLP-1 RA prescriptions in the first year. Second, given that maximal weight loss occurs around 6 months after GLP-1 RA initiation, ${ }^{7,9,10}$ we repeated the analyses after shortening the lag period to 6 months. Third, we assessed competing risk due to death from any cause by using the sub-distribution model proposed by Fine and Gray. ${ }^{33}$ Fourth, to account for potential time-dependent confounding across the weight loss categories during the follow-up period, we repeated the analyses using marginal structural modelling with inverse probability of treatment weighting, where covariates listed above were updated at 30day intervals over the follow-up period. ${ }^{34}$ Finally, we repeated the analyses after restricting the comparator group to DPP-4 inhibitors; these are incretin-based drugs that have not been previously associated with breast cancer. All analyses were conducted with SAS version 9.4 (SAS institute, Cary, NC) and R (R Foundation for Statistical Computing, Vienna, Austria). 


\section{RESULTS}

Overall, 173,115 female patients were newly-treated with non-insulin antidiabetic drugs between 1 January 2007 and 31 January 2018 (eFigure 2). Among these patients, 5,510 new users of GLP-1 RAs were matched to 5,510 new users of comparator drugs (eFigure 3). Both exposure groups were followed for a median (Q1, Q3) of $2.4(1.0,4.7)$ years.

Table 1 presents baseline characteristics of the matched exposure groups (see eTable $\mathbf{1}$ for the pre-matching characteristics); these were generally well balanced, with the exception of BMI and duration of treated diabetes which generated standardized differences of 0.10 . As a result, the outcome models were additionally adjusted for these variables. Among GLP-1 RA users, 65 patients were newly-diagnosed with breast cancer during 16,617 person-years of follow-up, compared with 50 patients during 16,583 person-years of followup in the comparator group (incidence rates of 3.9 [95\% CI: 3.0, 4.9] and 3.0 [95\% CI: 2.2, 3.2] per 1000 person-years, respectively).

During the follow-up period, the overall mammography screening rate was slightly higher among GLP-1 RA users, compared with comparator drug users (21.3 [95\% CI: 20.7, 21.9] v 20.2 [95\% CI: 19.6, 20.8] per 100 person-years, respectively). This mammography screening rate increased across maximal weight loss categories, with the highest rate among those losing $>10 \%$ of their weight (22.6 [95\% CI: 21.4-23.8] per 100 person-years) (eTable 2). Similarly, the incidence rate for referrals for diagnostic imaging or signs and symptoms of breast cancer was also higher among GLP-1 RA users, compared with comparator drug users (2.0 [95\% CI: 1.8, 2.2] v 1.9 [95\% CI: 1.7, 2.1] per 100 person-years, respectively). As with the mammography screening rates, the incidence rates also increased across weight loss categories (>10\% weight loss, 2.3 [95\% CI: 1.9, 2.7] per 100 person-years) (eTable 3).

Overall use of GLP-1 RAs was associated with an increased detection of breast cancer, generating a moderately elevated HR with a CI that included the null, when compared 
with use of comparator drugs (HR: $1.28,95 \%$ CI: $0.88,1.85)$. Similar results were observed in the as-treated analysis, which generated a more elevated HR with a CI that excluded the null (HR: 1.81, 95\% CI: 1.11, 2.96). For both intention-to-treat and as-treated approaches, GLP-1 RA users had a higher cumulative incidence of breast cancer, compared with comparator drug users; the curves both diverged at around 18 months after treatment initiation (eFigure 4 and eFigure 5, respectively).

Table 2 presents the results of the maximal weight loss analyses for the intention-totreat and as-treated approaches. In the intention-to-treat approach, breast cancer incidence gradually increased with GLP-1 RA maximal weight loss categories, with the highest HR observed for patients achieving at least $10 \%$ weight loss (HR: 1.77, 95\% CI: 1.12, 2.81). In the restricted cubic spline model, the 95\% CI of the HR excluded the null at a maximal weight loss of 9.8\%, and the highest HR was observed after achieving $15.4 \%$ weight loss (HR: 2.09, 95\% CI: 1.22, 3.58) (Figure 1). Similar results were observed in the as-treated analysis, but the HRs were slightly more elevated (>10\% weight loss, HR: 2.17, 95\% CI: $1.24,3.80)$.

\section{Secondary Analyses}

Figure 2 presents the GLP-1 RA maximal weight loss categories according to time since treatment initiation. In this analysis, the HR for $>10 \%$ maximal weight loss was elevated across all duration categories with overlapping CIs, although the highest HR was observed after 2 to 3 years since treatment initiation (HR: 2.90, 95\% CI: 1.21, 6.92). When stratifying by GLP-1 RA molecule, the HRs were higher for liraglutide than with exenatide, but both generated wide CIs due to few exposed events (eTable 4). Finally, when stratifying on BMI, morbidly obese patients tended to have a higher breast cancer incidence across all 
weight loss categories, particularly for $>10 \%$ maximal weight loss ( $p$-interaction $=0.66$ ) (eTable 5).

\section{Sensitivity Analyses}

The results of the sensitivity analyses are presented in eTables 6-10. First, re-defining the exposure by requiring at least four GLP-1 RA prescriptions in the first year did not materially change the HRs across weight loss categories. Second, shortening the exposure lag time from 1 year to 6 months led to increased HRs across weight loss categories. Third, results did not change when accounting for competing risk by death from any cause. Fourth, the marginal structural model generated HRs that were highly consistent with those of the primary analysis. Finally, restricting the comparator to DPP-4 inhibitors generated HRs that were consistent with those of the primary analysis, but generated wider CIs due to fewer exposed events. 


\section{DISCUSSION}

To our knowledge, this is the first observational study to investigate the weightlowering effects of GLP-1 RAs on the detection of breast cancer among patients with type 2 diabetes. In this study, the incidence of breast cancer gradually increased with GLP-1 RA maximal weight loss categories, with the highest incidence observed among those achieving at least $10 \%$ weight loss. Similar results were observed in both the intention-to-treat and astreated analyses, but with higher HRs in the latter, suggesting that breast cancer diagnoses occurred while patients were currently using GLP-1 RAs or shortly after. In secondary analyses, the highest incidence for those achieving $>10 \%$ weight loss was observed at 2 to 3 years since GLP-1 RA initiation.

Signals of a possible association between GLP-1 RAs and breast cancer were initially observed in premarketing trials of short durations (20 to 56 weeks). In weight management trials, an imbalance of breast cancer events was observed with liraglutide (3 mg daily dosing) versus placebo (17/2379 vs 3/1300, respectively). ${ }^{5}$ This imbalance was also observed in subsequent trials, such as the SCALE trial investigating the efficacy of liraglutide as a weight management treatment among obese patients without diabetes (10/2487 versus 3/1244, respectively). ${ }^{9}$ Of note, these events were observed relatively soon after randomization (within 56 weeks) among patients who had achieved at least $8 \%$ weight loss. ${ }^{9}$

A similar pattern was observed in trials assessing the efficacy of GLP-1 RAs in patients with type 2 diabetes. ${ }^{6,7,10}$ While there was no overall imbalance in breast cancer events with liraglutide (1.8 mg daily dosing) versus placebo in the LEADER trial (21/1657 versus 20/1680, respectively; HR: 1.06, 95\% CI: 0.57, 1.96), ${ }^{6}$ a post hoc analysis revealed a transient imbalance of breast cancer events with liraglutide between 1.5 to 2.5 years after randomization. ${ }^{10}$ This transient imbalance was also observed in our study, as well as in two observational studies that have assessed this association. ${ }^{11,12}$ More recently, an imbalance of 
breast cancer events was observed with semaglutide versus placebo in the SUSTAIN-6 trial, but this was based on few events (5/1648 versus 3/1649, respectively; HR: 1.73, 95\% CI: $0.41,7.34) .^{7}$

The mechanism through which GLP-1 RA-induced weight loss might improve the detection of breast masses is not well understood. However, there is evidence suggesting that obese women are less likely to engage in cancer screening programs, including mammography. ${ }^{9,17-20,35,36}$ As a result, this high risk population ${ }^{37}$ may be more likely to have prevalent disease. ${ }^{38}$ Thus, rapid and significant weight loss may lead to a better detection of prevalent breast masses, while also increasing the uptake and accuracy of breast examinations. ${ }^{17}$ Our findings support this hypothesis, as the mammography screening rate was higher across GLP-1 RA maximal weight loss categories, compared with the comparator. A similar pattern was also observed for the rate of referrals for diagnostic imaging and signs and symptoms of breast cancer (including breast masses). Finally, the relatively rapid timing of these events (within 2 to 3 years after treatment initiation) is consistent with a transient over-detection of breast cancer events among GLP-1 RA users.

This study has several strengths. First, weight measurements were readily available in the CPRD, which allowed us to model maximal weight loss with GLP-1 RAs. Second, we used a sequential propensity score-matched cohort design, which served to minimize residual confounding. Finally, in order to ensure a clinically-representative comparator group and further minimize residual confounding, we restricted the cohort to obese patients, as GLP-1 RAs are primarily prescribed to this population (92\% as reported in a recent CPRD study). ${ }^{11}$

This study has some limitations. First, as recorded weight measurements are dependent upon physician visits, maximal weight loss for some patients may have been subject to misclassification. Second, misclassification of exposure is possible, as the CPRD captures prescriptions written by general practitioners and not those written by specialists. 
However, chronic conditions such as type 2 diabetes are almost entirely managed in the primary care setting in the UK. ${ }^{39}$ Reassuringly, a sensitivity analysis that required GLP-1 RA users to have four prescriptions in the first year to be considered exposed did not change our results. Third, misclassification of the outcome is also possible, however, breast cancer has been shown to be well recorded in the CPRD. ${ }^{25}$ Fourth, given the observational nature of this study, residual confounding remains a concern. However, several important potential confounders were included in the propensity score model, such as smoking and laboratory values, which are often missing in administrative databases. We also observed similar findings in the marginal structural model that accounted for potential time-dependent confounding. Fifth, breast density information was not available, which has been shown to potentially affect mammographic screening sensitivity. ${ }^{40}$ Finally, some secondary analyses generated wide CIs due to fewer exposed events, and thus these results should be interpreted with caution.

While there is evidence that weight loss is associated with a decreased risk of breast cancer in the long-term, ${ }^{41}$ the results of this population-based study suggest that significant weight loss with GLP-1 RAs may increase the detection of prevalent breast cancer among obese women in the relative short-term. A better understanding of this relationship is particularly important given imbalances in breast cancer events observed in trials of novel antidiabetic drugs associated with weight loss, such as SGLT-2 inhibitors. ${ }^{42}$ Thus, this study provides physicians and patients valuable clinical information on the safety of GLP-1 RAs and its potential role in improving the detection of breast cancer. 


\section{REFERENCES}

1. Garber AJ. Long-Acting Glucagon-Like Peptide 1 Receptor Agonists. Diabetes Care. 2011;34(Supplement 2):S279.

2. Holst JJ. The physiology of glucagon-like peptide 1. Physiological reviews. 2007;87(4):1409-1439.

3. Manigault KR, Thurston MM. Liraglutide: A Glucagon-Like Peptide-1 Agonist for Chronic Weight Management. The Consultant pharmacist : the journal of the American Society of Consultant Pharmacists. 2016;31(12):685-697.

4. Shah M, Vella A. Effects of GLP-1 on appetite and weight. Reviews in endocrine \& metabolic disorders. 2014;15(3):181-187.

5. FDA Briefing Document: Endocrinologic and Metabolic Drugs Advisory Committee Meeting (EMDAC). 2017;

https://www.fda.gov/downloads/advisorycommittees/committeesmeetingmaterials/dru gs/endocrinologicandmetabolicdrugsadvisorycommittee/ucm563334.pdf.

6. Marso SP, Daniels GH, Brown-Frandsen K, et al. Liraglutide and Cardiovascular Outcomes in Type 2 Diabetes. N Engl J Med. 2016;375(4):311-322.

7. Marso SP, Bain SC, Consoli A, et al. Semaglutide and Cardiovascular Outcomes in Patients with Type 2 Diabetes. The New England journal of medicine. 2016;375(19):1834-1844.

8. Husain M, Birkenfeld AL, Donsmark M, et al. Oral Semaglutide and Cardiovascular Outcomes in Patients with Type 2 Diabetes. The New England journal of medicine. 2019.

9. Pi-Sunyer X, Astrup A, Fujioka K, et al. A Randomized, Controlled Trial of $3.0 \mathrm{mg}$ of Liraglutide in Weight Management. The New England journal of medicine. 2015;373(1):11-22. 
10. Nauck MA, Jensen TJ, Rosenkilde C, Calanna S, Buse JB. Neoplasms Reported With Liraglutide or Placebo in People With Type 2 Diabetes: Results From the LEADER Randomized Trial. Diabetes Care. 2018.

11. Hicks BM, Yin H, Yu OHY, Pollak MN, Platt RW, Azoulay L. Glucagon-like peptide-1 analogues and risk of breast cancer in women with type 2 diabetes: population based cohort study using the UK Clinical Practice Research Datalink. BMJ (Clinical research ed). 2016;355.

12. Funch D, Mortimer K, Li L, et al. Is there an association between liraglutide use and female breast cancer in a real-world setting? Diabetes, metabolic syndrome and obesity : targets and therapy. 2018;11:791-806.

13. Tannheimer SL, Rehemtulla A, Ethier SP. Characterization of fibroblast growth factor receptor 2 overexpression in the human breast cancer cell line SUM-52PE. Breast cancer research : BCR. 2000;2(4):311-320.

14. Christensen RM, Juhl CR, Torekov SS. Benefit-Risk Assessment of Obesity Drugs: Focus on Glucagon-like Peptide-1 Receptor Agonists. Drug Safety. 2019.

15. Excellence NNIfHaC. Type 2 diabetes in adults: management. 2017; https://www.nice.org.uk/guidance/ng28/chapter/1-Recommendations - drugtreatment-2.

16. Hinnen D. Glucagon-Like Peptide 1 Receptor Agonists for Type 2 Diabetes. Diabetes Spectrum. 2017;30(3):202.

17. Elmore JG, Carney PA, Abraham LA, et al. The association between obesity and screening mammography accuracy. Archives of internal medicine. 2004;164(10):1140-1147.

18. Fontaine KR, Heo M, Allison DB. Body weight and cancer screening among women. Journal of women's health \& gender-based medicine. 2001;10(5):463-470. 
19. Friedman AM, Hemler JR, Rossetti E, Clemow LP, Ferrante JM. Obese Women's Barriers to Mammography and Pap Smear: The Possible Role of Personality. Obesity. 2012;20(8):1611-1617.

20. Maruthur NM, Bolen S, Brancati FL, Clark JM. Obesity and mammography: a systematic review and meta-analysis. Journal of general internal medicine. 2009;24(5):665-677.

21. Reeves MJ, Newcomb PA, Remington PL, Marcus PM, MacKenzie WR. Body mass and breast cancer. Relationship between method of detection and stage of disease. Cancer. 1996;77(2):301-307.

22. Wolf A, Dedman D, Campbell J, et al. Data resource profile: Clinical Practice Research Datalink (CPRD) Aurum. International journal of epidemiology. 2019.

23. Herrett E, Gallagher AM, Bhaskaran K, et al. Data Resource Profile: Clinical Practice Research Datalink (CPRD). International journal of epidemiology. 2015;44(3):827836.

24. Herrett E, Thomas SL, Schoonen WM, Smeeth L, Hall AJ. Validation and validity of diagnoses in the General Practice Research Database: a systematic review. British journal of clinical pharmacology. 2009;69(1):4-14.

25. Boggon R, van Staa TP, Chapman M, Gallagher AM, Hammad TA, Richards MA. Cancer recording and mortality in the General Practice Research Database and linked cancer registries. Pharmacoepidemiol Drug Saf. 2013;22(2):168-175.

26. Jick SS, Kaye JA, Vasilakis-Scaramozza C, et al. Validity of the general practice research database. Pharmacotherapy. 2003;23(5):686-689.

27. Jick H, Jick SS, Derby LE. Validation of information recorded on general practitioner based computerised data resource in the United Kingdom. BMJ (Clinical research ed). 1991;302(6779):766-768. 
28. Hernan MA, Alonso A, Logan R, et al. Observational studies analyzed like randomized experiments: an application to postmenopausal hormone therapy and coronary heart disease. Epidemiology. 2008;19(6):766-779.

29. Ross ME, Kreider AR, Huang YS, Matone M, Rubin DM, Localio AR. Propensity Score Methods for Analyzing Observational Data Like Randomized Experiments: Challenges and Solutions for Rare Outcomes and Exposures. American journal of epidemiology. 2015;181(12):989-995.

30. Suissa S, Moodie EE, Dell'Aniello S. Prevalent new-user cohort designs for comparative drug effect studies by time-conditional propensity scores. Pharmacoepidemiol Drug Saf. 2017;26(4):459-468.

31. Durrleman S, Simon R. Flexible regression models with cubic splines. Stat Med. 1989;8(5):551-561.

32. Greenland S. Dose-response and trend analysis in epidemiology: alternatives to categorical analysis. Epidemiology. 1995;6(4):356-365.

33. Fine JP, Gray RJ. A Proportional Hazards Model for the Subdistribution of a Competing Risk. Journal of the American Statistical Association. 1999;94(446):496509.

34. Robins JM, Hernan MA, Brumback B. Marginal structural models and causal inference in epidemiology. Epidemiology. 2000;11(5):550-560.

35. Hernandez-Boussard T, Ahmed SM, Morton JM. Obesity disparities in preventive care: findings from the National Ambulatory Medical Care Survey, 2005-2007. Obesity (Silver Spring, Md). 2012;20(8):1639-1644.

36. Njor SH, von Euler-Chelpin M, Tjonneland A, Vejborg I, Lynge E. Body weight and sensitivity of screening mammography. European journal of cancer (Oxford, England : 1990). 2016;60:93-100. 
37. Field AE, Coakley EH, Must A, et al. Impact of overweight on the risk of developing common chronic diseases during a 10-year period. Archives of internal medicine. 2001;161(13):1581-1586.

38. Ferrante JM, Chen P-H, Crabtree BF, Wartenberg D. Cancer screening in women: body mass index and adherence to physician recommendations. Am J Prev Med. 2007;32(6):525-531.

39. Sharma M, Nazareth I, Petersen I. Trends in incidence, prevalence and prescribing in type 2 diabetes mellitus between 2000 and 2013 in primary care: a retrospective cohort study. BMJ Open. 2016;6(1):e010210.

40. Partain N, Mokdad A, Puzziferri N, et al. Mammographic density changes in surgical weight loss-an indication for personalized screening. BMC Med Imaging. 2018;18(1):10-10.

41. Chlebowski RT, Luo J, Anderson GL, et al. Weight loss and breast cancer incidence in postmenopausal women. Cancer. 2018.

42. Perkovic V, Jardine MJ, Neal B, et al. Canagliflozin and Renal Outcomes in Type 2 Diabetes and Nephropathy. New England Journal of Medicine. 2019. 


\section{FIGURE LEGENDS}

Figure 1. Restricted cubic spline of breast cancer associated with glucagon-like peptide-1 receptor agonist maximal weight loss.

Figure 2. Breast cancer associated with glucagon-like peptide-1 receptor agonist maximal weight loss categories according to time since treatment initiation.

Abbreviations: HR, hazard ratio; CI, confidence interval. 